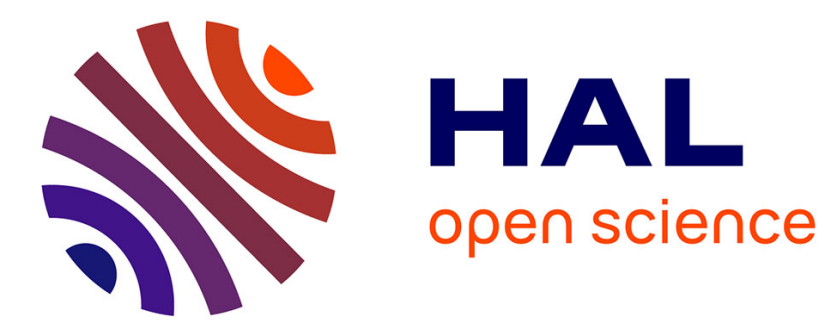

\title{
PANORAMA: A 3D Shape Descriptor based on Panoramic Views for Unsupervised 3D Object Retrieval
}

Panagiotis Papadakis, Ioannis Pratikakis, Theoharis Theoharis, Stavros

Perantonis

\section{- To cite this version:}

Panagiotis Papadakis, Ioannis Pratikakis, Theoharis Theoharis, Stavros Perantonis. PANORAMA: A 3D Shape Descriptor based on Panoramic Views for Unsupervised 3D Object Retrieval. International Journal of Computer Vision, 2010, 10.1007/s11263-009-0281-6 . hal-00758978

\section{HAL Id: hal-00758978 \\ https://hal.inria.fr/hal-00758978}

Submitted on 30 Nov 2012

HAL is a multi-disciplinary open access archive for the deposit and dissemination of scientific research documents, whether they are published or not. The documents may come from teaching and research institutions in France or abroad, or from public or private research centers.
L'archive ouverte pluridisciplinaire HAL, est destinée au dépôt et à la diffusion de documents scientifiques de niveau recherche, publiés ou non, émanant des établissements d'enseignement et de recherche français ou étrangers, des laboratoires publics ou privés. 


\title{
PANORAMA: A 3D Shape Descriptor based on Panoramic Views for Unsupervised 3D Object Retrieval
}

\author{
Panagiotis Papadakis - Ioannis Pratikakis - Theoharis Theoharis • \\ Stavros Perantonis
}

Received: date / Accepted: date

\begin{abstract}
We present a novel 3D shape descriptor that uses a set of panoramic views of a $3 \mathrm{D}$ object which describe the position and orientation of the object's surface in $3 \mathrm{D}$ space. We obtain a panoramic view of a $3 \mathrm{D}$ object by projecting it to the lateral surface of a cylinder parallel to one of its three principal axes and centered at the centroid of the object. The object is projected to three perpendicular cylinders, each one aligned with one of its principal axes in order to capture the global shape of the object. For each projection we compute the corresponding 2D Discrete Fourier Transform as well as 2D Discrete Wavelet Transform. We further increase the retrieval performance by employing a local (unsupervised) relevance feedback technique that shifts the descriptor of an object closer to its cluster centroid in feature space. The effectiveness of the proposed 3D object retrieval methodology is demonstrated via an extensive evaluation in standard benchmarks that clearly shows better performance against state-of-the-art 3D object retrieval methods.
\end{abstract}

Keywords 3D object retrieval - cylindrical projection . panorama local relevance feedback · unsupervised

Panagiotis Papadakis · Ioannis Pratikakis - Stavros Perantonis Computational Intelligence Laboratory, Institute of Informatics and Telecommunications, National Center for Scientific Research "Demokritos", Greece

E-mail: \{ppapadak,ipratika,sper\}@iit.demokritos.gr

Theoharis Theoharis

Computer Graphics Laboratory, Department of Informatics \&

Telecommunications, University of Athens, Greece

E-mail: theotheo@di.uoa.gr

\section{Introduction}

Content-based 3D object retrieval is an active research field that has attracted a significant amount of interest in recent years. This is due to the increasing availability of $3 \mathrm{D}$ objects through public or proprietary databases, as even novice users can create 3D models from scratch with easy to use modeling interfaces such as Teddy [1], Google SketchUp [2], ShapeShop [3] and others [4]. Applications such as CAD, computer games development, archaeology, bioinformatics, etc. are also playing a major role in the proliferation of $3 \mathrm{D}$ objects. A second source of $3 \mathrm{D}$ objects is $3 \mathrm{D}$ scanners, which are constantly gaining users as their price drops.

Already, 3D object search engines have been developed for commercial or research purposes that offer searching using 3D object queries or keyword based queries. The former approach, which is an instance of content-based retrieval (CBR) alleviates various limitations that are encountered in keyword based retrieval which become prohibitive as the number of $3 \mathrm{D}$ objects increases.

In CBR, each 3D object is represented by a shapedescriptor which is used to measure the similarity between two objects. The shape descriptor should capture the discriminative features of a 3D model, have a compact size and permit fast extraction and comparison time.

In this paper, we propose a novel 3D shape descriptor that exhibits top performance by using a set of features which are extracted from a set of panoramic views of a 3D object. The proposed descriptor is called PANORAMA which stands for PANoramic Object Representation for Accurate Model Attributing. The panoramic views are used to capture the position of the model's surface in 3D space as well as its orientation. 
We obtain a panoramic view of a 3D object by projecting it to the lateral surface of a cylinder aligned with one of the object's three principal axes and centered at the centroid of the object. The object's principal axes are determined during the rotation normalization step. The object is projected onto three perpendicular cylinders, each one aligned with one of its principal axes in order to capture the global shape of the object. For each projection, we compute the corresponding 2D Discrete Fourier Transform as well as 2D Discrete Wavelet Transform. To further enhance the retrieval performance, we employ a local relevance feedback (LRF) technique [5] that shifts the descriptor of an object closer to its cluster centroid in feature space. The employed LRF technique is unsupervised and assumes that the $k$ nearest neighbors of an object belong to the same class, without requiring any feedback from the user. We demonstrate the increased performance of the PANORAMA descriptor by comparing it to other stateof-the-art approaches in standard benchmarks and promote the application of the LRF technique by showing the performance gain that is obtained when it is combined with the PANORAMA descriptor.

The remainder of the paper is organized as follows: In Section 2, we provide an overview of the related work in the area of content-based 3D object retrieval (Section 2.1) and cite previous work that incorporates relevance feedback (Section 2.2). In Section 3, we give the detailed description for the extraction of the proposed 3D shape descriptor and in Section 4, we describe the procedure of the employed LRF technique. The results of an extensive evaluation of the proposed methodology are presented in Section 5 and conclusions are discussed in Section 6.

\section{Related work}

\subsection{Shape descriptors}

In this Section we provide an overview of the related work in the area of 3D shape descriptors for generic 3D object searching. 3D object retrieval methodologies that rely on supervision are beyond the scope of this review, since this paper focuses mainly on enhancing the effectiveness of retrieval by using discriminative shape features in an unsupervised context.

Content-based 3D object retrieval methods may be classified into two main categories according to the spatial dimensionality of the information used, namely 2D, $3 \mathrm{D}$ and their combination. In the following Sections, we review the state-of-the-art for each category.

\subsubsection{Methods based on 2D representations}

In this category, shape descriptors are generated from images-projections which may be contours, silhouettes, depth buffers or other kinds of 2D representations. Thus, similarity is measured using 2D shape matching techniques. Surprisingly, extended state-of-the-art reviews such as [6] and [7] show that descriptors belonging to this class exhibit better overall retrieval performance compared to descriptors that belong to the second class.

Chen et al. [8] proposed the Light Field descriptor, which is comprised of Zernike moments and Fourier coefficients computed on a set of projections taken from the vertices of a dodecahedron. Vranic [9] proposed a shape descriptor where features are extracted from depth buffers produced by six projections of the object, one for each side of a cube which encloses the object. In the same work, the Silhouette-based descriptor is proposed which uses the silhouettes produced by the three projections taken from the Cartesian planes. In [10], Passalis et al. proposed PTK, a depth buffer based descriptor which uses parallel projections to capture the object's thickness and an alignment scheme that is based on symmetry. Shih et al. [11] proposed the elevation descriptor where six depth buffers (elevations) are computed from the faces of the 3D object's bounding box and each buffer is described by a set of concentric circular areas that give the sum of pixel values within the respective areas. Ohbuchi et al. [12] proposed the Multiple Orientation Depth Fourier Transform (MODFT) descriptor where the model is projected from 42 viewpoints to cover all possible view aspects. Each depth buffer is then transformed to the $r-\theta$ domain and the Fourier transform is applied. To compare two objects, all possible pairs of coefficients are compared which inevitably increases comparison time. Zarpalas et al. [13] introduced a 3D shape descriptor called the spherical trace transform, which is the generalization of the $2 \mathrm{D}$ trace transform. In this method, a variety of $2 \mathrm{D}$ features are computed for a set of planes intersecting the volume of a 3D model. A newly proposed method is the depth line descriptor proposed by Chaouch et. al [14] where a 3D object is projected to the faces of its bounding box giving 6 depth buffers. Each depth buffer is then decomposed into a set of horizontal and vertical depth lines that are converted to state sequences which describe the change in depth at neighboring pixels.

\subsubsection{Methods based on 3D representations}

In this category, shape descriptors are extracted from $3 \mathrm{D}$ shape representations and the similarity is measured 
using appropriate representations in the spatial domain or in the spectral domain. A set of subcategories can be identified here, namely, statistical, graph-based and spherical function-based descriptors.

Statistical descriptors use histograms to capture the distributions of shape features. They are compact and fast to compute but they have very limited discrimination ability since they fail to capture local details that are characteristic of the object's shape. In the Shape Histograms descriptor proposed by Ankerst et al. [15], $3 \mathrm{D}$ space is divided into concentric shells, sectors, or both and for each part, the model's shape distribution is computed giving a sum of histograms bins. The shape distributions descriptor proposed by Osada et al. [16, 17] measures a set of shape characteristics for a random set of points belonging to the object, using appropriate shape functions, e.g. the D2 function which measures the distance between two random surface points. Ohbuchi et al. [18] proposed enhanced shape functions, namely the (absolute) angle distance histogram for inconsistently oriented meshes, which are extensions of the D2 shape distribution. Zaharia et al. [19] presented the $3 \mathrm{D}$ shape spectrum descriptor which is the histogram that describes the angular representation of the first and second principal curvature along the surface of the 3D object. In [20] Koertgen et al. make use of $3 \mathrm{D}$ shape contexts which are histograms each one corresponding to a surface point and capturing the distribution of the relative coordinates of the remaining surface points.

Graph-based methods use hierarchical structures to represent $3 \mathrm{D}$ objects and the similarity is measured using graph-matching techniques. These methods are suited to intra-class search, i.e. searching within very similar objects at different poses (articulations) but they have limited discrimination ability in generic object retrieval. Hilaga et al. [21] introduced the multi-resolution Reeb graph, which represents a 3D objects topology and skeletal structure at various levels of detail. In [22] Zhang et al. consider the use of medial surfaces to compute an equivalent directed acyclic graph of an object. In the work of Sundar et al. [23], the 3D object passes through a thinning process producing a set of skeletal points, which finally form a directed acyclic graph by applying the minimum spanning tree algorithm. Cornea et al. [24] propose the use of curve skeletons produced by the application of the generalized distance field to the volume of the $3 \mathrm{D}$ object and similarity is measured using the earth mover's distance. The P3DS descriptor developed by Kim et al. [25] uses an attributed relational graph whose nodes correspond to parts of the object that are represented using ellipsoids and the similarity is computed by employing the earth mover's distance.

A plurality of methods exists that use spherical functions to parameterize the shape of a 3D object. These methods exhibit good discrimination ability in general but most of them cannot capture shape features uniformly. This happens when the longitude-latitude parameterization is adopted that results in non-uniform sampling between the poles of the spherical function. Vranic [9] proposed the Ray-based descriptor which characterizes a 3D object by a spherical extent function capturing the furthest intersection points of the model's surface with rays emanating from the origin. Spherical harmonics or moments can be used to represent the spherical extent function. A generalization of the previous approach [9] uses several spherical extent functions of different radii. The GEDT descriptor proposed by Kazhdan et al. [26] is a volumetric representation of the Gaussian Euclidean Distance Transform of a 3D object, expressed by norms of spherical harmonic frequencies. In [27], the CRSP descriptor was proposed which uses the Continuous PCA (CPCA) along with Normals PCA (NPCA) to alleviate the rotation invariance problem and describes a 3D object using a volumetric sphericalfunction based representation expressed by spherical harmonics. Yu et al. [28] used spherical functions to describe the topology and concavity of the surface of a 3D object and the amount of effort required to transform it to its bounding sphere. Generalizing from 2D to 3D, Novotni et al. [29] presented the 3D Zernike descriptor, Daras et al. [30] introduced the generalized radon transform, Ricard et al. [31] developed the 3D ART descriptor by generalizing the $2 \mathrm{D}$ angular radial transform and Zaharia et al. [32] proposed the C3DHTD descriptor by generalizing the 2D Hough Transform.

\subsubsection{Hybrid methods}

Besides the previous categories, combinations of different methods have been considered in order to enhance the overall performance, which comprise a third category.

Vranic developed a hybrid descriptor called DESIRE [33], that consists of the Silhouette, Ray and Depth buffer based descriptors, which are combined linearly by fixed weights. The approach of Bustos et al. [34] assumes that the classification of a particular dataset is given, in order to estimate the expected performance of the individual shape descriptors for the submitted query and automatically weigh the contribution of each method. However, in the general case, the classification of a $3 \mathrm{D}$ model dataset is not fixed since the content of a $3 \mathrm{D}$ model dataset is not static. In the context of 
partial shape matching, Funkhouser et al. [35] use the predicted distinction performance of a set of descriptors based on a preceeding training stage and perform a priority driven search in the space of feature correspondences to determine the best match of features between a pair of models. The disadvantages of this approach is its time complexity which is prohibitive for online interaction as well as the storage requirements for the descriptors of all the models in the database. Based on the idea of combining features obtained from 2D and 3D representations, Song et al. [36] developed a descriptor that described an object by obtaining a set of orthogonal projections from different viewpoints and by measuring the curvature of the object's surface. Similar in spirit, Papadakis et al. [37] proposed a hybrid descriptor formed by combining features extracted from a depth-buffer and spherical-function based representation, with enhanced translation and rotation invariance properties. The advantage of this method over similar approaches is the top discriminative power along with minimum space and time requirements.

\subsection{Relevance Feedback in 3D object retrieval}

In order to enable the machine to retrieve information through adapting to individual categorization criteria, relevance feedback $(\mathrm{RF})$ was introduced as a means to involve the user in the retrieval process and guide the retrieval system towards the target. Relevance feedback was first used to improve text retrieval [38], later on successfully employed in image retrieval systems and lately in a few 3D object retrieval systems. It is the information that is acquired from the user's interaction with the retrieval system about the relevance of a subset of the retrieved results. Further information on relevance feedback methods can be found in [39], [40], [41] and $[5]$.

Local relevance feedback (LRF), also known as pseudo or blind relevance feedback, is different from the conventional approach in that the user does not actually provide any feedback at all. Instead, the required training data are obtained based only on the unsupervised retrieval result. The procedure comprises two steps. First, the user submits a query to the system which uses a set of low-level features to produce a ranked list of results which is not displayed to the user. Second, the system reconfigures itself by only using the top m matches of the list, based on the assumption that most likely they are relevant to the user's query.

LRF was first employed in the context of text retrieval, in order to extend the keywords comprising the query with related words from the top ranked retrieved documents. Apart from a few studies that incorporated
RF in 3D object retrieval [42],[43],[44],[45],[46],[47],[48], LRF has only lately been examined in [5].

\section{Computation of the PANORAMA descriptor}

In this Section, we first describe the steps for the computation of the proposed descriptor (PANORAMA), namely: (i) pose normalization (Section 3.1), (ii) extraction of the panoramic views (Section 3.2) and (iii) feature extraction (Section 3.3). Finally, in Section 3.4 we describe a weighing scheme that is applied to the features and the procedure for comparing two PANORAMA descriptors.

\subsection{Pose normalization}

Prior to the extraction of the PANORAMA descriptor, we must first normalize the pose of a $3 \mathrm{D}$ object, since the translation, rotation and scale characteristics should not influence the measure of similarity between objects.

To normalize the translation of a 3D model we compute its centroid using CPCA [9]. In CPCA, the centroid of a 3D mesh model is computed as the average of its triangle centroids where every triangle is weighed proportionally to its surface area. We translate the model so that its centroid coincides with the origin and translation invariance is achieved as the centroids of all $3 \mathrm{D}$ models coincide.

To normalize for rotation, we use CPCA and NPCA [27] in order to align the principal axes of a 3D model with the coordinate axes. First, we align the 3D model using CPCA to determine its principal axes using the model's surface area distribution and then we use NPCA to determine its principal axes using the surface orientation distribution. Both methods use Principal Component Analysis (PCA) to compute the principal axes of the 3D model. The difference between the two methods lies in the input data that are used for the computation of the covariance matrix. In particular, in CPCA the surface area coordinates are used whereas in NPCA the surface orientation coordinates are used which are obtained from the triangles' normal vectors. The full detailed description regarding the formulation of CPCA and NPCA can be found in [9] and in our previous work [27], respectively.

Thus, we obtain two alternative aligned versions of the 3D model, which are separately used to extract two sets of features that are integrated into a single feature vector (see Section 3.4).

The PANORAMA shape descriptor is rendered scale invariant, by normalizing the corresponding features to 
the unit $L_{1}$ norm. As will be later described in Sections 3.3.1 and 3.3.2, the features used by the PANORAMA descriptor are obtained from the 2D Discrete Fourier Transform and 2D Discrete Wavelet Transform. The respective coefficients are proportional to the object's scale, therefore by normalizing the coefficients to their unit $L_{1}$ norm we are in fact normalizing all objects to the same scale.

\subsection{Extraction of panoramic views}

After the normalization of a 3D model's pose, the next step is to acquire a set of panoramic views.

To obtain a panoramic view, we project the model to the lateral surface of a cylinder of radius $\mathrm{R}$ and height $H=2 R$, centered at the origin with its axis parallel to one of the coordinate axes (see figure 1). We set the value of $\mathrm{R}$ to $3 * d_{\text {mean }}$ where $d_{\text {mean }}$ is the mean distance of the model's surface from its centroid. For each model, the value of $d_{\text {mean }}$ is determined using the diagonal elements of the covariance matrix used in CPCA that give the average distances of the model's surface from the coordinate axes. Setting $R=3 * d_{\text {mean }}$ does not imply that a $3 \mathrm{D}$ model will necessarily lie completely inside the cylinder. However, this approach is better than using a bounding cylinder, that may contain the model in small scale in the presence of outlying parts of the model. The empirical value $3 * d_{\text {mean }}$ enables the majority of 3D models to lie completely inside the cylinder, while having a suitable scale to produce a descriptive projection.

In the following, we parameterize the lateral surface of the cylinder using a set of points $s(\varphi, y)$ where $\varphi \in[0,2 \pi]$ is the angle in the xy plane, $y \in[0, H]$ and we sample the $\varphi$ and $y$ coordinates at rates $2 B$ and

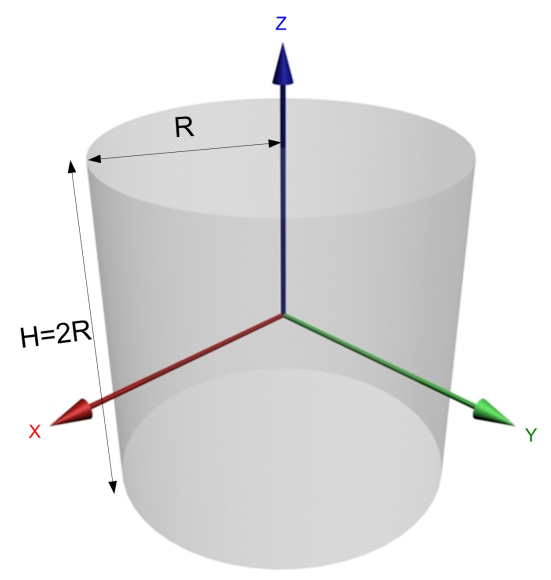

Fig. 1 The cylinder used for acquiring the projection of a $3 \mathrm{D}$ model.

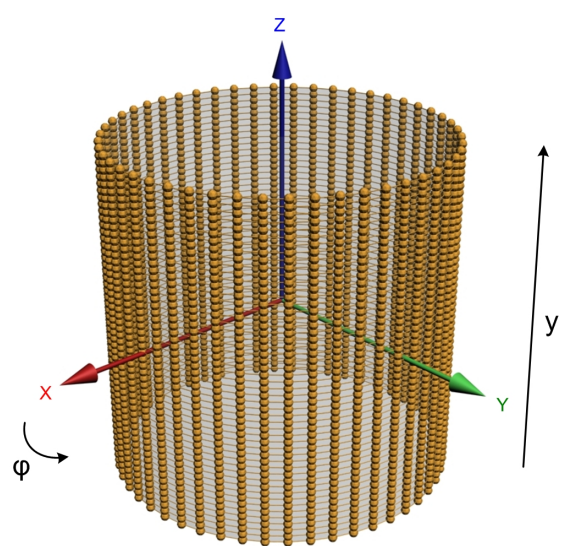

Fig. 2 The discretization of the lateral surface of the projection cylinder (points in orange) to the set of points $s\left(\varphi_{u}, y_{v}\right)$.

$B$, respectively (we set $B=64$ ). We sample the $\varphi$ dimension at twice the rate of the $y$ dimension to account for the difference in length between the perimeter of the cylinder's lateral surface and its height. Although the perimeter of the cylinder's lateral surface is $\pi \simeq 3$ times its height, we set the sampling rates at $2 B$ and $B$ respectively, since it was experimentally found as the best trade-off between effectiveness and efficiency. Thus we obtain the set of points $s\left(\varphi_{u}, y_{v}\right)$ where $\varphi_{u}=u * 2 \pi /(2 B), y_{v}=v * H / B, u \in[0,2 B-1]$ and $v \in[0, B-1]$. These points are shown in figure 2 .

The next step is to determine the value at each point $s\left(\varphi_{u}, y_{v}\right)$. The computation is carried out iteratively for $v=0,1, \ldots, B-1$, each time considering the coplanar $s\left(\varphi_{u}, y_{v}\right)$ points i.e. a cross-section of the cylinder at height $y_{v}$ and for each cross-section we cast rays from its center $c_{v}$ in the $\varphi_{u}$ directions. In figure 3 we show the points $s\left(\varphi_{u}, y_{v}\right)$ of the top-most cross-section $(v=B-$ 1 ) of the projection cylinder along with the respective rays emanating from the center of the cross-section.

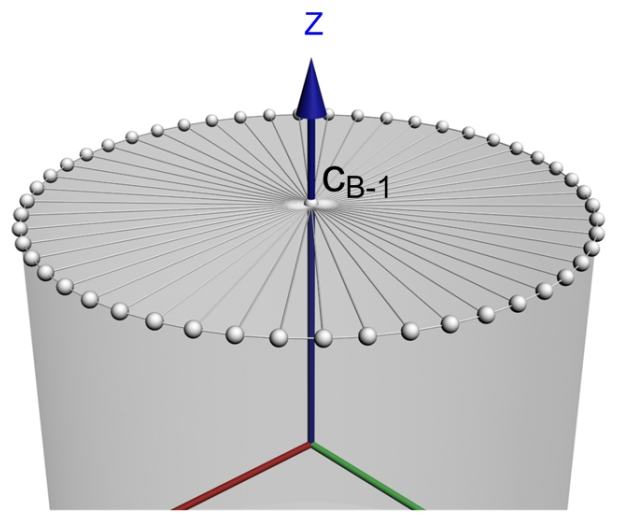

Fig. 3 The top-most cross section of the cylinder along with the corresponding rays emanating from the center of the cross-section $c_{B-1}$. 
The cylindrical projections are used to capture two characteristics of a 3D model's surface; (i) the position of the model's surface in 3D space and (ii) the orientation of the model's surface. To capture these characteristics we use two kinds of cylindrical projections $s_{1}\left(\varphi_{u}, y_{v}\right)$ and $s_{2}\left(\varphi_{u}, y_{v}\right)$. By default, the value of a point $s_{k}\left(\varphi_{u}, y_{v}\right), k \in\{1,2\}$ is set to zero.

To capture the position of the model's surface, for each cross-section at height $y_{v}$ we compute the distances from $c_{v}$ of the intersections of the model's surface with the rays at each direction $\varphi_{u}$.

Let $\operatorname{pos}\left(\varphi_{u}, y_{v}\right)$ denote the distance of the furthest from $c_{v}$ point of intersection between the ray emanating from $c_{v}$ in the $\varphi_{u}$ direction and the model's surface, then:

$s_{1}\left(\varphi_{u}, y_{v}\right)=\operatorname{pos}\left(\varphi_{u}, y_{v}\right)$

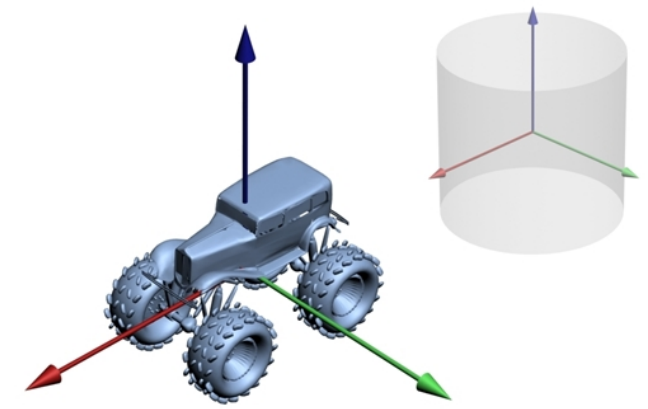

(a)

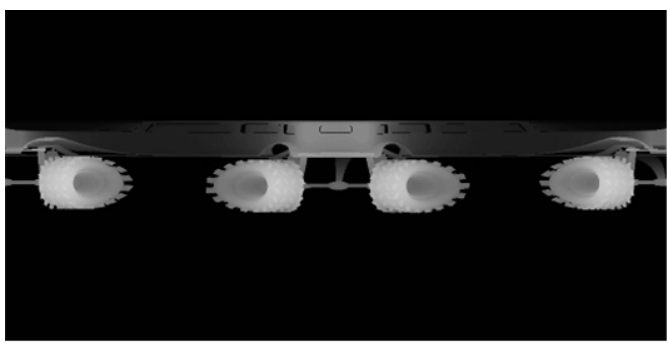

(b)

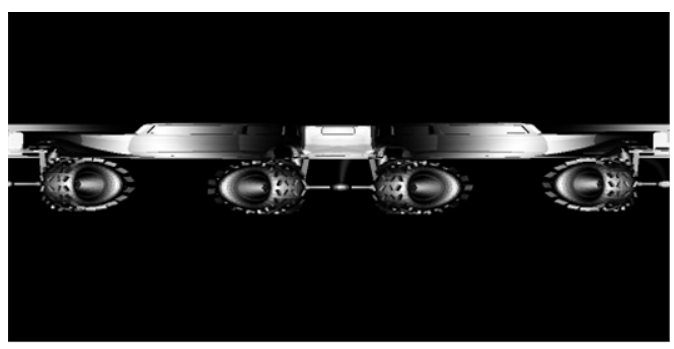

(c)

Fig. 4 (a) Pose normalized 3D model, (b) the unfolded cylindrical projection of the 3D model capturing the position of the surface (c) the unfolded cylindrical projection of the 3D model capturing the orientation of the surface.
Thus the value of a point $s_{1}\left(\varphi_{u}, y_{v}\right)$ lies in the range $[0, R]$, where $R$ denotes the radius of the cylinder.

A cylindrical projection can be viewed as a $2 \mathrm{D}$ grayscale image where pixels correspond to the $s_{k}\left(\varphi_{u}, y_{v}\right)$ intersection points and their values are mapped to the [black, white] space in a manner reminiscent to cylindrical texture mapping, see [49]. In figure 4 (a), we show an example 3D model along with a projection cylinder aligned with the z-axis. In figure 4 (b) the unfolded visual representation of its respective cylindrical projection $s_{1}\left(\varphi_{u}, y_{v}\right)$ is given.

To capture the orientation of the model's surface, for each cross-section at height $y_{v}$ we compute the intersections of the model's surface with the rays at each direction $\varphi_{u}$ and measure the angle between a ray and the normal vector of the triangle that is intersected. To determine the value of a point $s_{2}\left(\varphi_{u}, y_{v}\right)$ we use the cosine of the angle between the ray and the normal vector of the furthest from $c_{v}$ intersected triangle of the model's surface.

Let $\operatorname{ang}\left(\varphi_{u}, y_{v}\right)$ denote the above angle then the values of the $s_{2}\left(\varphi_{u}, y_{v}\right)$ points are given by:

$s_{2}\left(\varphi_{u}, y_{v}\right)=\left|\cos \left(\operatorname{ang}\left(\varphi_{u}, y_{v}\right)\right)\right|^{n}$

In figure 4 (c) the unfolded visual representation of the cylindrical projection $s_{2}\left(\varphi_{u}, y_{v}\right)$ is given for the model shown in figure 4 (a).

We take the $n^{\text {th }}$ power of $\left|\cos \left(\operatorname{ang}\left(\varphi_{u}, y_{v}\right)\right)\right|$, where $n \geq 2$, since this setting enhances the contrast of the produced cylindrical projection which was experimentally found to be more discriminative. Setting $\mathrm{n}$ to any value within $[4,6]$ gave the best results while taking the absolute value of the cosine is necessary to deal with inconsistently oriented triangles along the object's surface. We do not enhance the contrast of the $s_{1}\left(\varphi_{u}, y_{v}\right)$ projection since it was found to produce less discriminative features.

Although the cylindrical projection captures a large part of the shape of a model, a single cylindrical projection may not be able to capture concave parts. In figure 5 we show a typical example of this. Figure 5 (a) shows the 3D model of a cup and figure 5 (b) shows the produced cylindrical projection when using a projection cylinder that is aligned with the $\mathrm{z}$ axis. As can be observed, this projection cannot capture the interior part of the cup.

To alleviate this problem, we compute cylindrical projections from differently oriented cylinders, i.e. cylinders that are aligned with all coordinate axes in order to obtain a more informative 3D model representation. Thus, we project a $3 \mathrm{D}$ model to the lateral surfaces of three cylinders, each one aligned with a different coordinate axis as shown in figure 5 (b)-(d), which produces three sets of points $s_{k, t}\left(\varphi_{u}, y_{v}\right)$ for $k \in\{1,2\}$, 


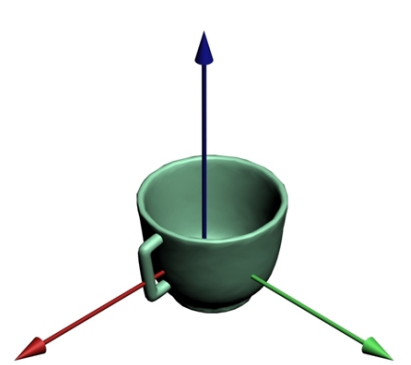

(a)

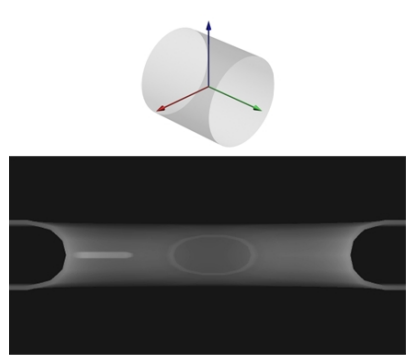

(c)

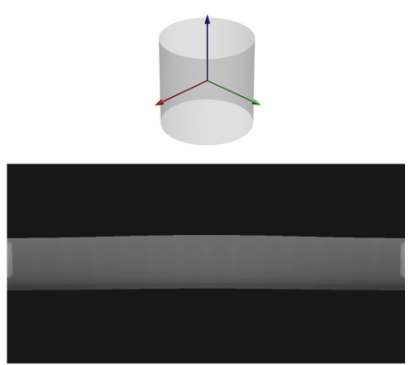

(b)

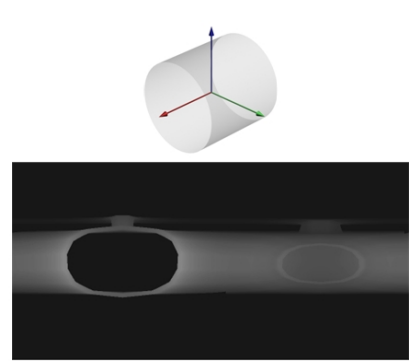

(d)
Fig. 5 (a) A 3D model of a cup and (b)-(d) the corresponding cylindrical projections $s_{1, t}\left(\varphi_{u}, y_{v}\right)$ using three cylinders each one aligned with the $\mathrm{z}, \mathrm{y}$ and $\mathrm{x}$ coordinate axis respectively.

where $t \in\{x, y, z\}$ denotes the axis that the cylinder is aligned with. For the cylindrical projections that are aligned with the $\mathrm{x}$ and $\mathrm{y}$ axes, the $\varphi_{u}$ variable is measured at the yz and zx planes respectively while all other notations remain the same.

\subsection{Feature representation of panoramic views}

In this Section, we detail the procedure for the generation of a set of features that describe a panoramic view. Toward this goal, we use the 2D Discrete Fourier Transform (Section 3.3.1) and 2D Discrete Wavelet Transform (Section 3.3.2).

\subsubsection{D Discrete Fourier Transform}

For each cylindrical projection $s_{k, t}\left(\varphi_{u}, y_{v}\right)(k \in\{1,2\}$ and $t \in\{x, y, z\})$, we compute the corresponding $2 \mathrm{D}$ Discrete Fourier Transform (DFT), which is given by:

$F_{k, t}(m, n)=\sum_{u=0}^{2 B-1} \sum_{v=0}^{B-1} s_{k, t}\left(\varphi_{u}, y_{v}\right) \cdot e^{-2 j \pi\left(\frac{m u}{2 B}+\frac{n v}{B}\right)}$

where $m \in[0,2 B-1]$ and $n \in[0, B-1]$.

Since $s_{k, t}\left(\varphi_{u}, y_{v}\right)$ is a real-valued function, the Hermitian symmetry property holds for the Fourier coefficients, i.e. $F_{k, t}(u, v)=F_{k, t}^{*}(2 B-u, B-v)$, where $F^{*}$ denotes the complex conjugate. Hence, the size of the non-redundant information is a set of $\left(\frac{2 B}{2}+1\right) *\left(\frac{B}{2}+1\right)$

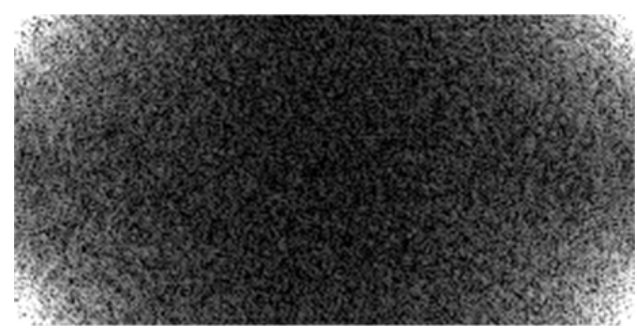

(a)

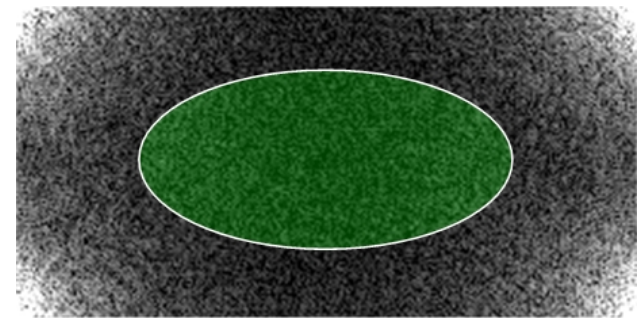

(b)

Fig. 6 (a) A typical 2D Fourier transform of a cylindrical projection; (b) The Fourier coefficients that lie inside the green-colored area are discarded to reduce dimensionality.

Fourier coefficients for each projection. Next, we store the absolute values of the real and imaginary part of each coefficient and normalize the coefficients to the unit $L_{1}$ norm, which ensures scaling invariance as explained in Section 3.1.

In practice, most of the energy of the Fourier coefficients is concentrated on the four corners of the image of the transform, as can be seen in figure 6 (a). Therefore, we only keep a subset of the full set of Fourier coefficients, i.e. those containing most of the energy. This can be straightforwardly done by considering an ellipse positioned at the center of the Fourier image and discarding all the coefficients that lie on the interior of the ellipse, as shown in figure 6 (b). The ratio of the width to the height of the ellipse is equal to the ratio of the width to the height of the Fourier image and the size of the ellipse is set so that the low energy coefficients that are discarded correspond to approximately $35 \%$ of the total number of coefficients per projection.

After the completion of all previous operations, the resulting coefficients are denoted by $\tilde{F}_{k, t}$. Thus, the final feature set $\mathbf{s}_{F}$ of Fourier coefficients for a particular aligned version of a $3 \mathrm{D}$ object is denoted by:

$\mathbf{s}_{F}=\left(\tilde{F}_{1, x}, \tilde{F}_{2, x}, \tilde{F}_{1, y}, \tilde{F}_{2, y}, \tilde{F}_{1, z}, \tilde{F}_{2, z}\right)$

\subsubsection{D Discrete Wavelet Transform}

For each cylindrical projection $s_{k, t}\left(\varphi_{u}, y_{v}\right)(k \in\{1,2\}$ and $t \in\{x, y, z\})$, we compute the corresponding $2 \mathrm{D}$ Discrete Wavelet Transform (DWT), which is given by: 


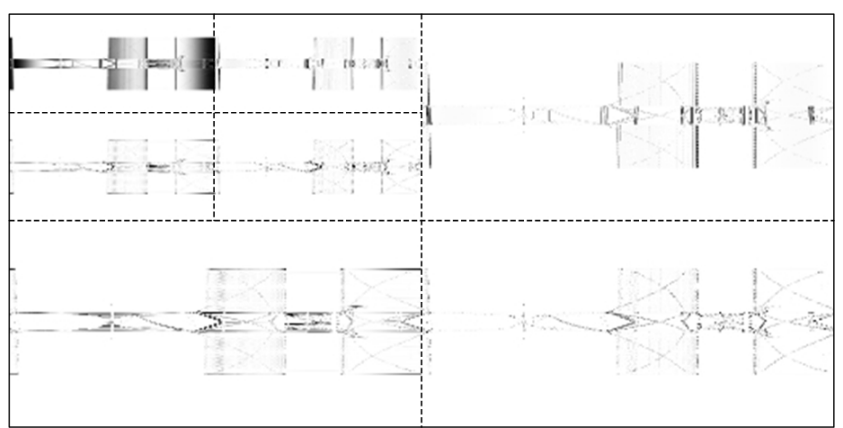

Fig. 7 2-level wavelet transformation of a cylindrical projection of an airplane (the image is shown in negative colors).

$$
\begin{aligned}
& W_{k, t}^{\varphi}\left(j_{0}, m, n\right)= \\
& \frac{1}{\sqrt{2 B \cdot B}} \cdot \sum_{u=0}^{2 B-1} \sum_{v=0}^{B-1} s_{k, t}\left(\varphi_{u}, y_{v}\right) \cdot \varphi_{j_{0}, m, n}(u, v) \\
& W_{k, t}^{\psi}(j, m, n)= \\
& \frac{1}{\sqrt{2 B \cdot B}} \cdot \sum_{u=0}^{2 B-1} \sum_{v=0}^{B-1} s_{k, t}\left(\varphi_{u}, y_{v}\right) \cdot \psi_{j, m, n}(u, v)
\end{aligned}
$$

where $m \in[0,2 B-1], n \in[0, B-1], j \geq j_{0}$ is the scale of the multi-level DWT, $j_{0}$ is the starting scale and $\varphi_{j_{0}, m, n}(u, v), \psi_{j, m, n}(u, v)$ is the scaling and wavelet function respectively. The $W_{k, t}^{\varphi}\left(j_{0}, m, n\right)$ approximationscaling coefficients correspond to the low-pass subband of the transform at the starting scale $j_{0}$. The $W_{k, t}^{\psi}(j, m, n)$ detail-wavelet coefficients correspond to the vertical, horizontal and diagonal subbands. We take the absolute values of the coefficients and normalize to their $L_{1}$ norm, which are now denoted as $\tilde{W}_{k, t}^{\varphi}\left(j_{0}, m, n\right)$ and $\tilde{W}_{k, t}^{\psi}(j, m, n)$.

In figure 7 , we show the image of a 2 -level wavelet transformation of a cylindrical projection. The transform is shown in negative colors to better point out the detail coefficients.

In our implementation, we computed the DWT of each cylindrical projection up to the last level. In particular, since the dimensions of a cylindrical projection are $(2 B) \cdot(B)$, the total number of levels of the DWT are $\log _{2} B$ and thus $j=0,1, \ldots \log _{2} B-1$.

To compute the DWT we have used the Haar and Coiflet (C6) filters (basis functions), as they attained the best overall performance. Therefore, two distinctive DWTs are computed, the first using the Haar and the second using the Coiflet basis functions.

Instead of using directly the coefficients of the DWT as shape features, we compute a set of standard statistic image features that are listed below:

\section{i. Mean}

$$
\mu=\frac{1}{n_{x} n_{y}} \sum_{x=1}^{n_{x}} \sum_{y=1}^{n_{y}} I(x, y)
$$

ii. Standard deviation

$$
\sigma=\sqrt{\frac{1}{n_{x} n_{y}} \sum_{x=1}^{n_{x}} \sum_{y=1}^{n_{y}}(I(x, y)-\mu)^{2}}
$$

\section{iii. Skewness}

$$
\beta=\frac{\frac{1}{n_{x} n_{y}} \sum_{x=1}^{n_{x}} \sum_{y=1}^{n_{y}}(I(x, y)-\mu)^{3}}{\sigma^{3}}
$$

where $n_{x}, n_{y}$ are the dimensions of an image $\mathrm{I}(\mathrm{x}, \mathrm{y})$.

In our case, the above features are computed for each sub-image of the DWT, i.e. for each subband in every level of the DWT. Therefore, $I(x, y)$ is replaced by the $\tilde{W}_{k, t}^{\varphi}\left(j_{0}, m, n\right)$ and $\tilde{W}_{k, t}^{\psi}(j, m, n)$ coefficients accordingly. Since we have $\log _{2} B$ levels in the DWT, the total number of subbands per cylindrical projection is $\left(3 * \log _{2} B+1\right)$. Hence, since we use two wavelet basis functions and three statistic image features, we obtain a total of $2 * 3 *\left(3 * \log _{2} B+1\right)$ features per cylindrical projection which are denoted as $\tilde{V}_{k, t}$ and the final feature set $\mathbf{s}_{W}$ for a particular aligned version of a $3 \mathrm{D}$ object is denoted as:

$\mathbf{s}_{W}=\left(\tilde{V}_{1, x}, \tilde{V}_{2, x}, \tilde{V}_{1, y}, \tilde{V}_{2, y}, \tilde{V}_{1, z}, \tilde{V}_{2, z}\right)$

\subsection{Features weighing and matching}

The features that were generated for each panoramic view are weighed by a factor $w_{t}$, according to the orientation of the cylinder ( $\mathrm{x}, \mathrm{y}$ or $\mathrm{z}$ ) that was used to acquire the cylindrical projection. We apply this weighing based on the observation that not all cylindrical projections capture the same amount of information about the model's shape. The t-projection cylinder that is parallel to the $t$ coordinate axis corresponds to one of the principal axes of the $3 \mathrm{D}$ model that were determined in the rotation normalization step. The amount of information that is captured by the t-cylindrical projection is directly related to the principal axes of the model's surface that are encoded, as is demonstrated in figure 8 for an example 3D model of a race car. In this example, the first, second and third principal axis of the object's surface is aligned with the $\mathrm{x}, \mathrm{y}$ and $\mathrm{z}$ axis respectively, therefore the most informative cylindrical projection corresponds to the $\mathrm{x}$-projection cylinder (figure 8 (d)), followed by the y-projection cylinder (figure 8 (c)) and the least informative z-projection cylinder (figure 8 (b)). We set the factors $w_{t}$ to fixed values that were determined experimentally as $w_{x}=0.51, w_{y}=0.31$ and $w_{z}=0.18$. 


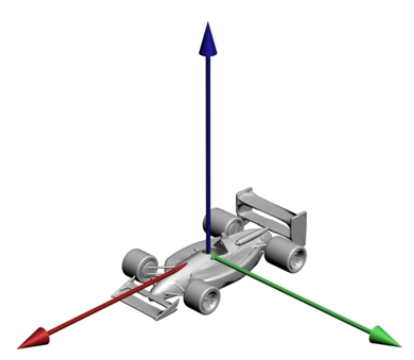

(a)

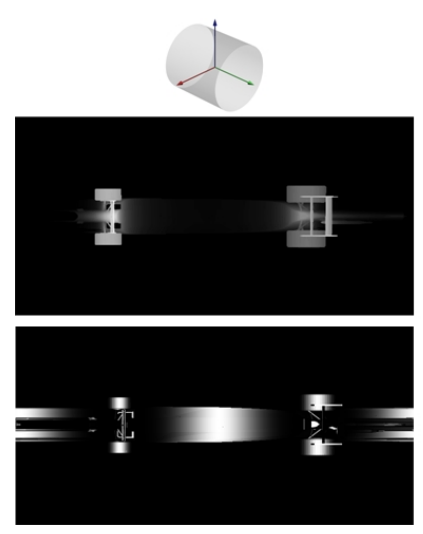

(c)

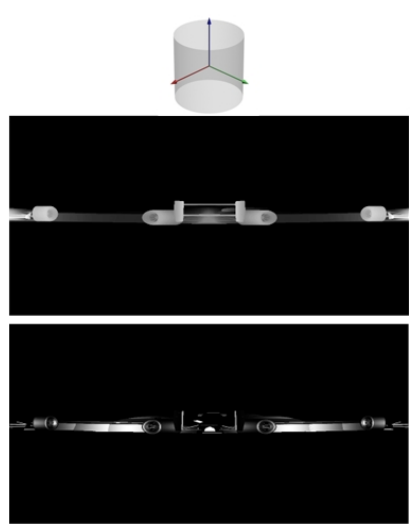

(b)

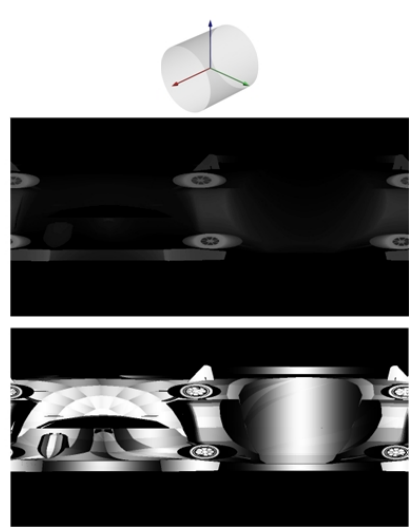

(d)
Fig. 8 (a) A 3D model of a car and (b)-(d) the respective cylindrical projections $s_{1, t}\left(\varphi_{u}, y_{v}\right)$ and $s_{2, t}\left(\varphi_{u}, y_{v}\right)$ for $t=z, t=$ $y$ and $t=x$ respectively.

To compute the distance between the same aligned version (CPCA or NPCA) of two 3D objects, we compute the distances between the corresponding sets of features. We denote the features set of a particular aligned version of an object by:

$\mathbf{p}_{l}=\left(\mathbf{s}_{F, l}, \mathbf{s}_{W, l}\right), l \in\{c p c a, n p c a\}$

and the full PANORAMA descriptor of 3D object by:

$\mathbf{P}=\left(\mathbf{p}_{c p c a}, \mathbf{p}_{n p c a}\right)$

Considering the features sets $\mathbf{p}_{l}, \dot{\mathbf{p}}_{l}$ of two $3 \mathrm{D}$ objects of the same aligned version $l$, the distance is computed by:

$d_{l}\left(\mathbf{p}_{l}, \dot{\mathbf{p}}_{l}\right)=L_{1}\left(\mathbf{s}_{F, l}, \dot{\mathbf{s}}_{F, l}\right)+D_{c a n}\left(\mathbf{s}_{W, l}, \dot{\mathbf{s}}_{W, l}\right)$

where $L_{1}(),, D_{\text {can }}($,$) denotes the Manhattan and the$ Canberra distance [50] respectively, each one normalized to the $[0,1]$ space.

The overall similarity between two $3 \mathrm{D}$ objects is measured by computing the distance between the sets of features of the same aligned version and the comparison that gives the minimum distance between the two alignments, sets the final score. Thus the overall distance $D(\mathbf{P}, \mathbf{P})$ between two PANORAMA descriptors $\mathbf{P}$ and $\mathbf{P}$ is given by:

$$
D(\mathbf{P}, \dot{\mathbf{P}})=\min _{l} d_{l}\left(\mathbf{p}_{l}, \dot{\mathbf{p}}_{l}\right)
$$

\section{Local Relevance Feedback (LRF) technique}

In this section, we outline the major points of the employed LRF method whose detailed description can be found in [5]. The method comprises two stages, the online and the off-line stage.

During the off-line stage, the descriptor of each stored object is updated using its $\mathrm{k}$ nearest neighbors in feature space. These are assumed to belong to the same class and the updated descriptor is the average of the original descriptor and a weighed centroid of the k nearest neighbors.

During the on-line stage, a user submits a query to the system which finds within the updated feature space its k nearest neighbors and updates its descriptor according to rule that was applied during the off-line stage. The updated descriptor of the query is then used to measure its similarity against every object of the database and display the results.

The relevance assumption for the $\mathrm{k}$ nearest neighbors is not always valid as irrelevant objects that may belong to the $\mathrm{k}$ nearest neighbors will be mistaken as relevant. This is known as query drift and implies the scenario where the retrieval system is misled by the irrelevant data and drawn away from the user's target. However, if the features that are used to compare two objects are discriminative enough to cluster most objects that belong to the same class, then the relevance assumption will be valid in the majority of cases and the average performance will be better.

In [5] it was shown that LRF increased the performance of the CRSP descriptor [27]. The PANORAMA descriptor is far more discriminative therefore reducing the negative effect of the query drift phenomenon and rendering LRF more applicable.

The number $\mathrm{k}$ of nearest neighbors that are considered as relevant to a query is determined by the expected recall of the employed shape features near the neighborhood of a query. In this case, setting $\mathrm{k}=4$ gave the best results which amounts to approximately $30 \%$ of the objects of a class on average, for the datasets used in Section 5. 


\section{Results}

We next present the results of an extensive evaluation of the proposed PANORAMA descriptor and LRF technique. We tested performance using the following standard 3D model datasets:

(i.) The classified dataset of CCCC [9].

(ii.) The classified dataset of the National Taiwan University Benchmark (NTU, http://3d.csie.ntu.edu.tw/).

(iii.) The dataset of the Watertight Models track of SHape REtrieval Contest 2007 (WM-SHREC) [51].

(iv.) The MPEG-7 dataset (http://www.chiariglione.org/mpeg/).

(v.) The test dataset of the Princeton Shape Benchmark (PSB) [6].

(vi.) The dataset of the Engineering Shape Benchmark (ESB) [52].

Table 1 gives the characteristics of these datasets.

Table 1. Characteristics of the evaluation datasets.

\begin{tabular}{|l|c|c|c|}
\hline Dataset & \#models & \#classes & type \\
\hline CCCC & 472 & 55 & generic \\
\hline NTU & 549 & 47 & generic \\
\hline WM-SHREC & 400 & 20 & generic \\
\hline MPEG-7 & 1300 & 135 & generic \\
\hline PSB & 907 & 92 & generic \\
\hline ESB & 866 & 48 & CAD \\
\hline
\end{tabular}

To evaluate the performance we use precision-recall diagrams. Recall is the ratio of relevant to the query retrieved models to the total number of relevant models while precision is the ratio of relevant to the query retrieved models to the number of retrieved models. The evaluations were performed by using each model of a dataset as a query in the remaining set of models and computing the average precision-recall performance overall models.

\subsection{Robustness}

In this Section we test the robustness of the PANORAMA descriptor under the presence of noise. We have experimented with various degrees of Gaussian noise added along the surface of each 3D object as shown in figure 9. Apparently, adding Gaussian noise with $\sigma>0.01$ has a destructive effect on the object's surface and most of the geometric details are lost. We believe that noisy $3 \mathrm{D}$ objects such as the ones shown in figure 9 (c)-(d) are rarely encountered and practically useless, thus we are more interested in the robustness of the PANORAMA descriptor with respect to levels of noise that compare to the examples shown in figure 9 (b).

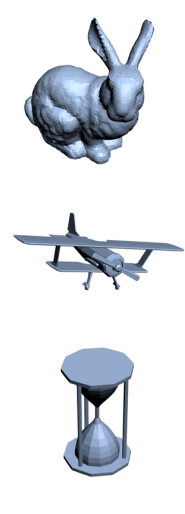

(a)
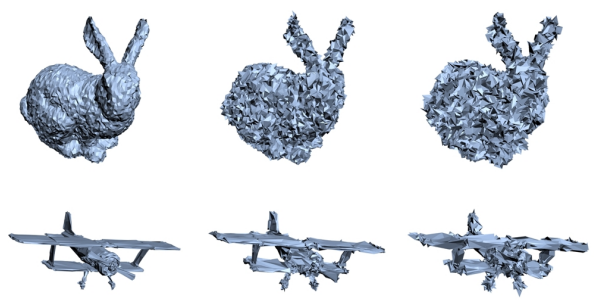

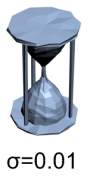

(b)

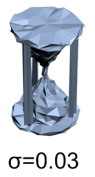

(c)

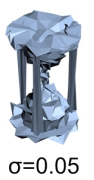

(d)
Fig. 9 Demonstration of the effect of different degrees of additive Gaussian noise on the surface of the original 3D object (a).

Since the PANORAMA descriptor is based on normalizing the rotation of a $3 \mathrm{D}$ object, we measured the effect of noise in the determination of the object's principal axes for CPCA and NPCA. The results of this experiment are shown in table 2 where we show the average angular perturbation of the object's principal axes after the addition of noise within the PSB dataset.

Table 2. Effect of noise in the determination of principal axes for the CPCA and NPCA rotation normalization methods within the PSB dataset.

\begin{tabular}{|l|c|c|c|}
\hline Alignment method & $\sigma=0.01$ & $\sigma=0.03$ & $\sigma=0.05$ \\
\hline CPCA & $6^{\circ}$ & $6.6^{\circ}$ & $7.4^{\circ}$ \\
\hline NPCA & $10.4^{\circ}$ & $14^{\circ}$ & $16.8^{\circ}$ \\
\hline
\end{tabular}

It is easy to understand that a certain change in the coordinates of the vertices that comprise a polygon has a greater relative impact on the orientation of the polygon's normal vector. This can be demonstrated in figure 10 where we show the depth buffers that are obtained after adding the same degrees of Gaussian noise for the bunny 3D object of figure 9 (a). Apparently, the effect of noise is more clearly noticed in figure 9 in contrast to figure 10 where only the depth is used. Thus, the fact that the NPCA alignment method is affected by noise to a greater degree than $\mathrm{CPCA}$ is a reasonable result. Hence, we can say that $\mathrm{CPCA}$ is more robust than NPCA with respect to noise.

Nevertheless, a perturbation of the object's principal axes after the addition of noise does not necessarily mean that the alignment of $3 \mathrm{D}$ objects is worse and lead to reduced performance in 3D object retrieval. For example, if the principal axes of 3D objects are perturbed toward the same directions for objects of the same class, then we expect to attain similar retrieval performance.

In the sequel, we evaluate the performance of the PANORAMA descriptor before and after the addition of different levels of Gaussian noise along the surface of 
(a)

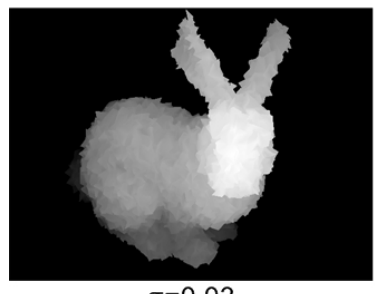

$\sigma=0.03$

(c)

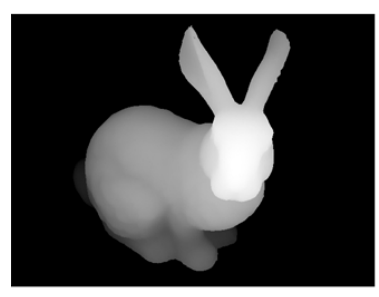

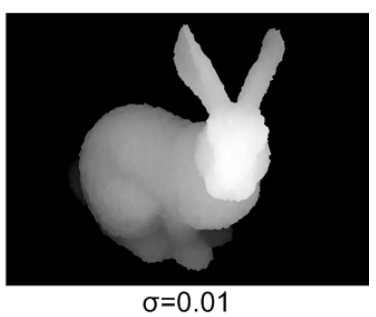

(b)

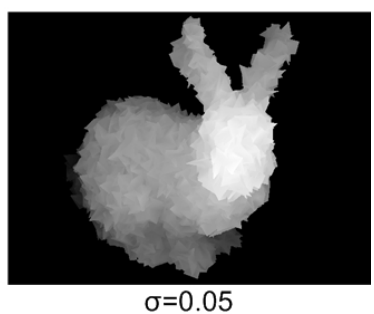

(d)
Fig. 10 Demonstration of the effect of different degrees of additive Gaussian noise on a depth buffer.

$3 \mathrm{D}$ objects as was performed previously. However, we should note that the performance of the descriptor is not only affected by the perturbed principal axes but also from the alternated surface of 3D objects after the addition of noise which would result in different cylindrical projections as well as features. In figure 11, we demonstrate the performance in terms of precision and recall within the PSB dataset. This experiment demonstrates that the PANORAMA descriptor is very robust with respect to reasonable levels of noise, as in the case where $\sigma=0.01$. In this case, we can see that the performance of PANORAMA is totally unaffected while for greater levels of noise $(\sigma=0.03$ and $\sigma=0.05)$ the performance decreases on a reasonable scale compared to the destructive effect of noise that is demonstrated in figure 9 .

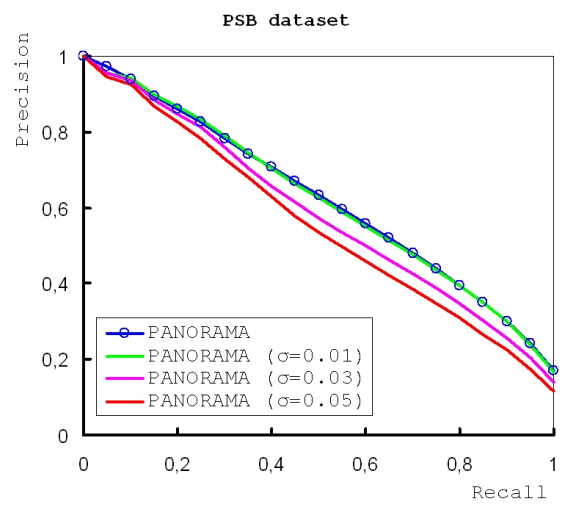

Fig. 11 Demonstration of the effect of different degrees of additive Gaussian noise on the retrieval performance of the PANORAMA descriptor.

\subsection{Justification of PANORAMA settings}

In this Section we demonstrate the increase in retrieval performnace that is achieved due to specific settings of the PANORAMA descriptor. In particular, in figure 12 (a) we compare the performance when using $s_{1, t}\left(\varphi_{u}, y_{v}\right), s_{2, t}\left(\varphi_{u}, y_{v}\right)$ or both and in figure 9 (b) we evaluate the performance between using a single and using three perpendicular cylinders aligned with the coordinate axes. The results clearly show that a significant increase in discriminative power is attained when capturing both the surface depth and orientation as well as using three perpendicular cylinders instead of one.

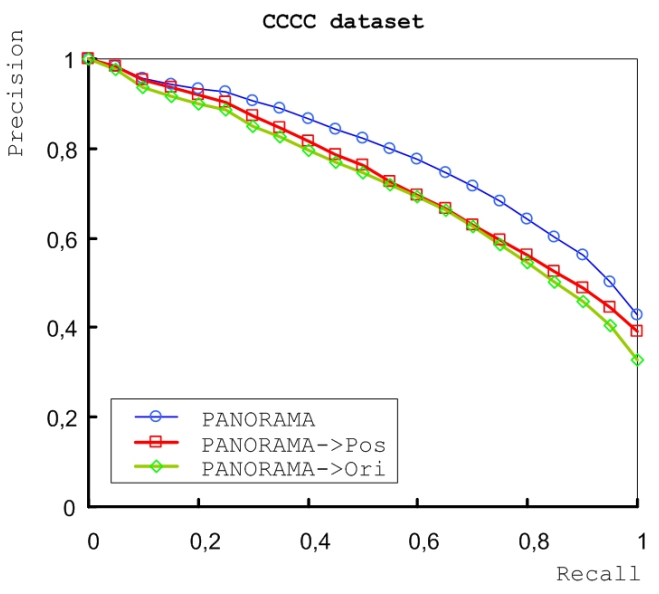

(a)

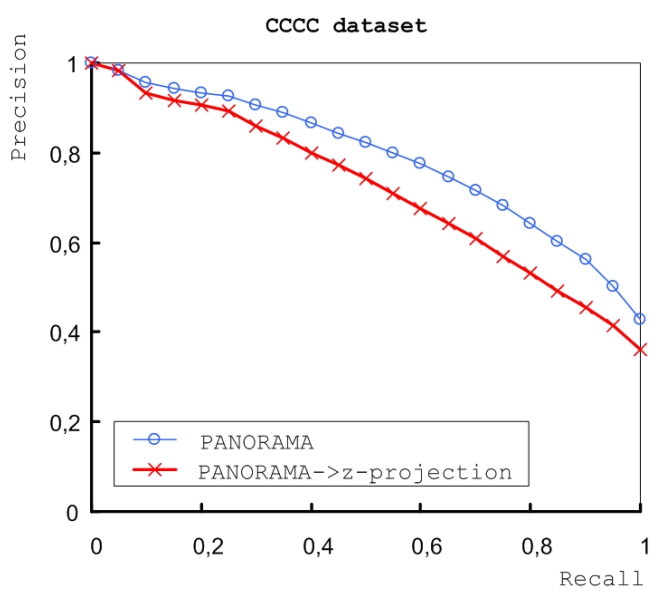

(b)

Fig. 12 Performance evaluation of the PANORAMA descriptor when using: (a) $s_{1, t}\left(\varphi_{u}, y_{v}\right)(\operatorname{PANORAMA} \rightarrow \operatorname{Pos}), s_{2, t}\left(\varphi_{u}, y_{v}\right)$ (PANORAMA $\rightarrow$ Ori) or both (PANORAMA); (b) a single cylinder aligned with the $\mathrm{z}$ coordinate axis (PANORAMA $\rightarrow \mathrm{z}$ projection) and a set of three perpendicular cylinders aligned with the object's principal axes (PANORAMA). 

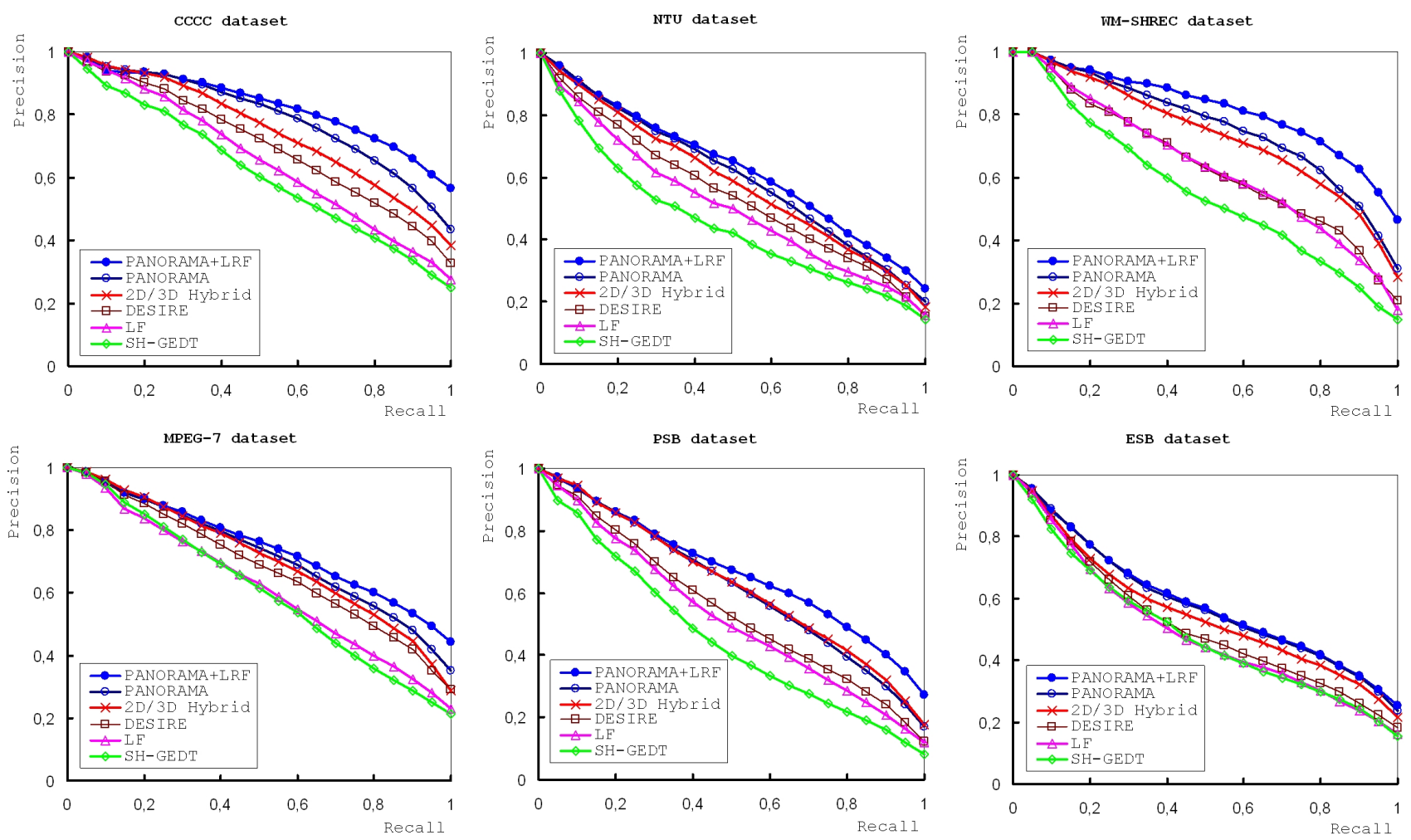

Fig. 13 Precision-recall plots comparing the proposed PANORAMA descriptor against the 2D/3D Hybrid, DESIRE, LF and SHGEDT descriptor in various 3D model datasets. The comparison includes the combination of the PANORAMA descriptor with local relevance feedback (LRF).

\subsection{Comparative Evaluation}

We next compare the PANORAMA descriptor against the following state-of-the-art methods:

- The 2D/3D Hybrid descriptor developed by Papadakis et al. [37].

- The DESIRE descriptor developed by Vranic et al. [33].

- The Light Field descriptor (LF) developed by Chen et al. [8].

- The spherical harmonic representation of the Gaussian Euclidean Distance Transform descriptor (SHGEDT) developed by Kazhdan et al. [26].

In figure 13, we give the precision-recall diagrams comparing the proposed PANORAMA descriptor against the other descriptors and show the increase in performance when LRF is employed. It is evident that the PANORAMA descriptor attains a better overall performance compared to the other methods. Interestingly, although the LF descriptor uses a plurality of 2D images (100 orthogonal projections) it is outperformed by the PANORAMA descriptor which uses a total of 12 cylindrical projections that are acquired from just three perpendicular cylinders. In addition, the PA-
NORAMA descriptor is more discriminative than the 2D/3D Hybrid descriptor which uses a total of 12 orthogonal projections combined with 48 spherical functions. This strongly suggests that the cylindrical projection is a more effective representation for the purpose of 3D object retrieval compared to the conventional orthogonal projection and spherical function based representation. This is also confirmed by comparing PANORAMA with the DESIRE descriptor that also uses 12 orthogonal projections (6 depth buffers and 6 silhouettes) as well as features extracted using a spherical function.

In addition, it is evident that LRF adds a major gain in the overall performance, particularly on the CCCC, WM-SHREC and PSB datasets. This indicates that the query drift phenomenon that comes with LRF is compensated for the increased precision of the PANORAMA descriptor within the top retrieved results. These results are coherent to those that were obtained using the CRSP descriptor in [5] and show that the employed LRF technique is general purpose and can be applied to any retrieval method as long as precision is high near the neighborhood of the query. It is also worth noticing that the LRF technique increases precision mainly at higher values of recall, as can be seen in 
figure 13. This implies that a user notices the increase in performance as he browses through the list of results until most or all models appear that belong to the query's class. It also implies that employing LRF more than once before showing the results to the user will not add any further gain in the retrieval performance. This is because the employed LRF technique uses the $\mathrm{k}$ nearest neighbors of a $3 \mathrm{D}$ model to update its descriptor but since precision is not increased at the early stages of recall, the $\mathrm{k}$ nearest neighbors of the model after employing LRF will mostly be the same as before employing LRF.

To further quantify the performance of the compared methods, we next employ the following measures:

- Nearest Neighbor (NN): The percentage of queries where the closest match belongs to the query's class.

- First Tier $(\mathrm{FT})$ : The recall for the $(C-1)$ closest matches were $C$ is the cardinality of the query's class.

- Second Tier (ST) : The recall for the $2(C-1)$ closest matches were $C$ is the cardinality of the query's class.

These measures range from $0 \%-100 \%$ and higher values indicate better performance. In table 2 , we give the scores of each method for each measure for all datasets.

Apparently, the PANORAMA descriptor consistently outperforms all compared methods with respect to these measures as well. We can also observe that the nearest neighbor score is not particularly affected by the application of LRF, compared to the first and second tier measures whose values are significantly increased after employing LRF. This confirms our earlier conclusion that LRF does not change the precision at the early stages of recall and therefore it does not add any further gain in performance if it is applied multiple times.

As described in Section 3.3, the PANORAMA descriptor uses two kinds of features, namely those coming from the $2 \mathrm{D} \operatorname{DFT}\left(\mathbf{s}_{F}\right)$ and the $2 \mathrm{D} \operatorname{DWT}\left(\mathbf{s}_{W}\right)$. Comparing the retrieval performance between the two kinds, the $\mathbf{s}_{F}$ component was found to be more effective in most datasets than the $\mathbf{s}_{W}$ part. In fact, the $\mathbf{s}_{W}$ part had a slight advantage in retrieval performance within the WM-SHREC and ESB datasets. Overall however, the $\mathbf{s}_{F}$ part is about $3 \%$ better in terms of average precision, which can be justified by the fact that $\mathbf{s}_{F}$ has greater dimensionality than the $\mathbf{s}_{W}$ part. In particular, the dimensionality of $\mathbf{s}_{F}$ is $2 *\left(\frac{2 B}{2}+1\right) *\left(\frac{B}{2}+1\right)$ while for the $\mathbf{s}_{W}$ component it is only $2 * 3 *\left(3 * \log _{2} B+1\right)$. Since we set the bandwidth to $B=64$, this amounts to a dimension of 4160 and 114 respectively. This implies, that we can attain comparable retrieval performance using just the $\mathbf{s}_{W}$ part of the full descriptor.

Table 2. Quantitative measures scores for the proposed PANORAMA, 2D/3D Hybrid, DESIRE, LF and SH-GEDT methods for the CCCC, NTU, WM-SHREC, $M P E G-7, P S B$ and ESB datasets.

\begin{tabular}{|l||c|c|c|}
\hline Method & NN $(\%)$ & FT $(\%)$ & ST $(\%)$ \\
\hline PANORAMA+LRF & 87.4 & $\mathbf{7 0 . 3}$ & $\mathbf{8 6 . 6}$ \\
PANORAMA & $\mathbf{8 7 . 9}$ & 66.3 & 81.2 \\
2D/3D Hybrid & 87.4 & 60.2 & 75.8 \\
DESIRE & 82.8 & 55.6 & 70.0 \\
LF & 79.8 & 50.2 & 63.1 \\
SH-GEDT & 75.7 & 45.9 & 59.9 \\
\hline
\end{tabular}

\begin{tabular}{|l||c|c|c|}
\hline Method & NN (\%) & FT (\%) & ST (\%) \\
\hline PANORAMA+LRF & $\mathbf{7 9 . 1}$ & $\mathbf{5 1 . 6}$ & $\mathbf{6 4 . 3}$ \\
PANORAMA & 78.6 & 49.0 & 61.5 \\
2D/3D Hybrid & 76.2 & 46.6 & 59.1 \\
DESIRE & 71.9 & 42.7 & 55.4 \\
LF & 70.0 & 39.0 & 50.1 \\
SH-GEDT & 58.8 & 33.9 & 46.3 \\
\hline
\end{tabular}

\begin{tabular}{|l||c|c|c|}
\hline \multicolumn{4}{c|}{ WM-SHREC } \\
\hline Method & NN (\%) & FT (\%) & ST (\%) \\
\hline PANORAMA+LRF & $\mathbf{9 5 . 7}$ & $\mathbf{7 4 . 3}$ & $\mathbf{8 3 . 9}$ \\
2D/3D Hybrid & 95.7 & 67.3 & 78.4 \\
DESIRE & 95.5 & 64.2 & 77.3 \\
LF & 91.7 & 53.5 & 67.3 \\
SH-GEDT & 92.3 & 52.6 & 66.2 \\
\hline \multicolumn{4}{|c|}{ MPEG-7 } \\
\hline
\end{tabular}

\begin{tabular}{|l||c|c|c|}
\hline Method & NN (\%) & FT (\%) & ST (\%) \\
\hline PANORAMA+LRF & $\mathbf{8 7 . 2}$ & $\mathbf{6 5 . 5}$ & $\mathbf{7 5 . 9}$ \\
PANORAMA & 87.2 & 61.8 & 73.1 \\
2D/3D Hybrid & 86.1 & 59.6 & 70.7 \\
DESIRE & 86.4 & 57.7 & 67.7 \\
LF & 80.2 & 51.7 & 61.9 \\
SH-GEDT & 83.7 & 50.3 & 59.4 \\
\hline
\end{tabular}

\begin{tabular}{|l||c|c|c|}
\hline Method & NN $(\%)$ & FT (\%) & ST (\%) \\
\hline PANORAMA+LRF & 75.2 & $\mathbf{5 3 . 1}$ & $\mathbf{6 5 . 9}$ \\
PANORAMA & $\mathbf{7 5 . 3}$ & 47.9 & 60.3 \\
2D/3D Hybrid & 74.2 & 47.3 & 60.6 \\
DESIRE & 65.8 & 40.4 & 51.3 \\
LF & 65.7 & 38.0 & 48.7 \\
SH-GEDT & 55.3 & 31.0 & 41.4 \\
\hline \multicolumn{4}{|c|}{ ESB } \\
\hline Method & NN (\%) & FT (\%) & ST $(\%)$ \\
\hline PANORAMA+LRF & $\mathbf{8 7 . 0}$ & $\mathbf{4 9 . 9}$ & $\mathbf{6 5 . 8}$ \\
PANORAMA & 86.5 & 49.4 & 64.1 \\
2D/3D Hybrid & 82.9 & 46.5 & 60.5 \\
DESIRE & 82.3 & 41.7 & 55.0 \\
LF & 82.0 & 40.4 & 53.9 \\
SH-GEDT & 80.3 & 40.1 & 53.6 \\
\hline
\end{tabular}




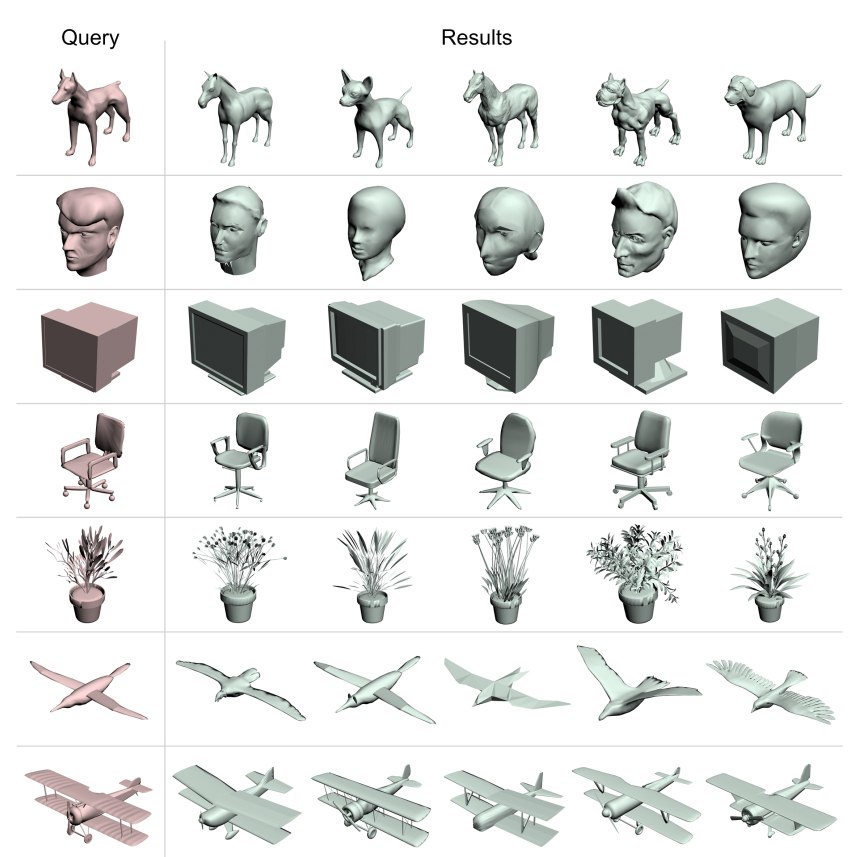

Fig. 14 Examples of queries within the PSB dataset and the respective top 5 retrieved models using the PANORAMA descriptor. The retrieved objects are ranked from left to right in decreasing order of similarity.

This is a significant advantage of the proposed method as it can be rendered much more efficient by reducing its storage requirements and time complexity, with negligible cost in discriminative power.

Altogether, the PANORAMA descriptor is very efficient in computation and comparison time. On a standard contemporary machine, it takes less than one second to extract and pairwise comparison time is approximately $0.23 \mathrm{~ms}$ which enables real-time retrieval from large repositories. These are the average values over the total set of models from the datasets used in our evaluation.

In figure 14, we provide a few examples of queries and the respective top 5 retrieved models from the PSB dataset using the proposed PANORAMA descriptor.

\section{Conclusions}

In this paper, we presented a novel 3D shape descriptor called PANORAMA, that allows effective contentbased 3D model retrieval exhibiting superior performance, compared to other state-of-the-art approaches. PANORAMA is based on a novel 3D shape representation that uses a set of panoramic views of the $3 \mathrm{D}$ model which are obtained by projecting the model to the lateral surfaces of a set of cylinders that are aligned with the model's principal axes. A panoramic view is particularly descriptive of an object's shape as it cap- tures a large portion of the object that would otherwise require multiple orthogonal projections from different viewpoints. It is also beneficial compared to spherical function based representations as the underlying sampling is uniform in the Euclidean space.

The 2-dimensional parameterization of a cylindrical projection allows the application of a variety of $2 \mathrm{D}$ features used for 2D shape-matching to be directly applied in the context of $3 \mathrm{D}$ shape matching. In this paper we have used the 2D Discrete Fourier Transform together together with the 2D Discrete Wavelet Transform and their combination enables very effective and efficient $3 \mathrm{D}$ object retrieval. Using only the wavelet features part of the descriptor, we can greatly increase efficiency in terms of storage requirements and time complexity, with negligible cost in discriminative power.

Moreover, we have used the PANORAMA descriptor to examine the application of local relevance feedback in the context of content-based $3 \mathrm{D}$ object retrieval, using the method that was proposed in [5]. The results of the evaluation showed that this method can add a considerable gain in the overall retrieval performance and can be readily applied to other retrieval methods that exhibit high precision near the neighborhood of the query.

\section{References}

1. Igarashi, T., Matsuoka, S., Tanaka, H.: Teddy: a sketching interface for 3D freeform design. In: SIGGRAPH '99: Proceedings of the 26th annual conference on computer graphics and interactive techniques. (1999) 409-416

2. Google SketchUp: http://sketchup.google.com/.

3. Schmidt, R., Wyvill, B., Sousa, M.C., Jorge, J.A.: Shapeshop: Sketch-based solid modeling with blobtrees. In: Proceedings of the 2nd Eurographics Workshop on SketchBased Interfaces and Modeling. (2005)

4. Olsen, L., Samavati, F., Sousa, M.C., Jorge, J.: A taxonomy of modeling techniques using sketch-based interfaces. In: Eurographics. (1999) 39-57

5. Papadakis, P., Pratikakis, I., Trafalis, T., Theoharis, T., Perantonis, S.: Relevance feedback in content-based 3d object retrieval: A comparative study. Computer-Aided Design and Applications 5(5) (2008) 753-763

6. Shilane, P., Min, P., Kazhdan, M., Funkhouser, T.: The princeton shape benchmark. In: Shape Modeling International. (2004) 167-178

7. Bustos, B., Keim, D., Saupe, D., Schreck, T., Vranić, D.V.: Feature-based similarity search in 3 d object databases. ACM Comput. Surv. 37(4) (2005) 345-387

8. Chen, D.Y., Tian, X.P., Shen, Y.T., Ouhyoung, M.: On visual similarity based 3D model retrieval. Eurographics, Computer Graphics Forum (2003) 223-232

9. Vranic, D.V.: 3D Model Retrieval. PhD thesis, University of Leipzig (2004)

10. Passalis, G., Theoharis, T., Kakadiaris, I.A.: Ptk: A novel depth buffer-based shape descriptor for three-dimensional object retrieval. Visual Computer 23(1) (2006) 5-14 
11. Shih, J.L., Lee, C.H., Wang, J.: A new 3D model retrieval approach based on the elevation descriptor. Pattern Recognition 40(1) (2007) 283-295

12. Ohbuchi, R., Nakazawa, M., Takei, T.: Retrieving 3D shapes based on their appearance. In: 5th ACM SIGMM international workshop on Multimedia information retrieval. (2003) $39-45$

13. Zarpalas, D., Daras, P., Axenopoulos, A., Tzovaras, D., Strintzis, M.G.: 3D model search and retrieval using the spherical trace transform. EURASIP Journal on Advances in Signal Processing (2007) Article ID 23912, 14 pages

14. Chaouch, M., Verroust-Blondet, A.: 3D model retrieval based on depth line descriptor. In: Multimedia and Expo, 2007 IEEE International Conference on. (2007) 599-602

15. Ankerst, M., Kastenmuller, G., Kriegel, H.P., Seidl, T.: Nearest neighbor classification in 3D protein databases. In: ISMB. (1999) 34-43

16. Osada, R., Funkhouser, T., Chazelle, B., Dobkin, D.: Matching 3D models with shape distributions. In: Int. Conf. on Shape Modeling and Applications. (2001) 154-166

17. Osada, R., Funkhouser, T., Chazelle, B., Dobkin, D.: Shape distributions. ACM Transactions on Graphics 21(4) (2002) 807-832

18. Ohbuchi, R., Minamitani, T., Takei, T.: Shape-similarity search of 3D models by using enhanced shape functions. Computer Applications in Technology 23(2-4) (2005) 70-85

19. Zaharia, T., Petreux, F.: 3D shape-based retrieval within the mpeg-7 framework. In: SPIE Conf. on Nonlinear Image Processing and Pattern Analysis XII. (2001) 133-145

20. Sundar, H., Silver, D., Gagvani, N., Dickinson, S.: 3D shape matching with 3D shape contexts. In: Seventh Central European Seminar on Computer Graphics. (2003)

21. Hilaga, M., Shinagawa, Y., Kohmura, T., Kunii, T.L.: Topology matching for fully automatic similarity estimation of 3D shapes. In: Proceedings of the 28th annual conference on Computer graphics and interactive techniques. (2001) 203212

22. Zhang, J., Siddiqi, K., Macrini, D., Shokoufandeh, A., Dickinson, S.: Retrieving articulated 3-d models using medial surfaces and their graph spectra. In: International Workshop On Energy Minimization Methods in Computer Vision and Pattern Recognition. (2005)

23. Sundar, H., Silver, D., Gagvani, N., Dickinson, S.: Skeleton based shape matching and retrieval. In: Proceedings of the Shape Modeling International. (2003) 130-139

24. Cornea, N., Demirci, M., Silver, D., Shokoufandeh, A., Dickinson, S., Kantor, P.: 3D object retrieval using many-to-many matching of curve skeletons. In: Proceedings of the International Conference on Shape Modeling and Applications. (2005) 368-373

25. Kim, D.H., Park, I.K., Yun, I.D., Lee, S.U.: A new mpeg-7 standard: Perceptual 3-d shape descriptor. In: Pacific Conference in Multimedia. (2004) 238-245

26. Kazhdan, M., Funkhouser, T., Rusinkiewicz, S.: Rotation invariant spherical harmonic representation of 3D shape descriptors. In: Eurographics/ACM SIGGRAPH symposium on Geometry processing. (2003) 156-164

27. Papadakis, P., Pratikakis, I., Perantonis, S., Theoharis, T.: Efficient 3D shape matching and retrieval using a concrete radialized spherical projection representation. Pattern Recognition 40(9) (2007) 2437-2452

28. Yu, M., Atmosukarto, I., Leow, W.K., Huang, Z., Xu, R.: 3D model retrieval with morphing-based geometric and topological feature maps. In: 5th ACM SIGMM International Workshop on Multimedia Information Retrieval. (2003) 656-661

29. Novotni, M., Klein, R.: 3d zernike descriptors for content based shape retrieval. In: 8th ACM symposium on Solid Modeling and Applications. (2003) 216-225
30. Daras, P., Zarpalas, D., Tzovaras, D., Strintzis, M.G.: Efficient 3-D model search and retrieval using generalized 3-D radon transforms. IEEE Transactions on Multimedia 8(1) (2006) 101-114

31. Ricard, J., Coeurjolly, D., Baskurt, A.: Generalization of angular radial transform for $2 \mathrm{D}$ and $3 \mathrm{D}$ shape retrieval. Pattern Recognition Letters 26 (2005) 2174-2186

32. Zaharia, T., Preteux., F.: Shape-based retrieval of 3D mesh models. (2002) 437-440

33. Vranic, D.V.: Desire: a composite 3D-shape descriptor. In: Proceedings of the IEEE International Conference on Multimedia and Expo

34. Bustos, B., Keim, D., Saupe, D., Schreck, T., Vranic, D. Automatic selection and combination of descriptors for effective 3D similarity search. In: IEEE Sixth Int. Symp. on Multimedia Software Engineering. (2004) 514-521

35. Funkhouser, T., Shilane, P.: Partial matching of 3D shapes with priority-driven search. In: Fourth Eurographics symposium on Geometry processing. (2006) 131-142

36. Song, J.J., Golshani, F.: Shape-based 3D model retrieval. In: Proceedings of the IEEE International Conference on Tools with Artificial Intelligence

37. Papadakis, P., Pratikakis, I., Theoharis, T., Passalis, G., Perantonis, S.: 3D object retrieval using an efficient and compact hybrid shape descriptor. In: Eurographics Workshop on 3D Object Retrieval. (2008)

38. Rochio, J.: 14. In: Relevance Feedback in Information Retrieval The SMART Retrieval System - Experiments in Automatic Document Processing. Prentice Hall (1971) 313-323

39. Ruthven, I., Lalmas, M.: A survey on the use of relevance feedback for information access systems. Knowl. Eng. Rev. 18(2) (2003) 95-145

40. Crucianu, M., Ferecatu, M., Boujemaa, N.: Relevance feedback for image retrieval: a short survey. Technical report, INRIA (2004)

41. Zhou, X.S., Huang, T.S.: Exploring the nature and variants of relevance feedback. In: Proceedings of the IEEE Workshop on Content-based Access of Image and Video Libraries. (2001) 94-101

42. Elad, M., Tal, A., Ar, S.: Content based retrieval of vrml objects: an iterative and interactive approach. In: Proceedings of the 6th Eurographics workshop on Multimedia. (2001)

43. Bang, H., Chen, T.: Feature space warping: An approach to relevance feedback. In: Proceedings of the Int. Conf. on Image Processing. (2002)

44. Atmosukarto, I., Kheng, W., Huang, Z.: Feature combination and relevance feedback for 3D model retrieval. In: Proceedings of the 11th Int. Conf. on Multimedia Modeling. (2005)

45. Lou, K., Jayanti, S., Iyer, N., Kalyanaraman, Y., Ramani, K.: A reconfigurable 3D engineering shape search system part ii: Database indexing, retrieval and clustering. In: Proceedings of the 23rd Computers and Information in Engineering Conference. (2003)

46. Leifman, G., Meir, R., Tal, A.: Semantic-oriented 3d shape retrieval using relevance feedback. Visual Computer 21(8-10) (2005) 865-875

47. Akbar, S., Kung, J., Wagner, R., Prihatmanto, A.S.: Multifeature integration with relevance feedback on 3D model similarity retrieval. In: Proceedings of the International Conference on Information Integration and Web-based Applications Services. (2006) 77-86

48. Novotni, M., Park, G.J., Wessel, R., Klein, R.: Evaluation of kernel based methods for relevance feedback in 3D shape retrieval. In: Proceedings of the 4th Int. Workshop on ContentBased Multimedia Indexing. (2005)

49. Theoharis, T., Papaioannou, G., Platis, N., Patrikalakis, N.: Graphics and Visualization: Principles and Algorithms. AK Peters, Wellesley, MA (2008) 
50. Kokare, M., Chatterji, B., Biswas, P.: Comparison of similarity metrics for texture image retrieval. In: TENCON 2003. Conference on Convergent Technologies for Asia-Pacific Region. Volume 2. (2003) 571-575

51. Veltkamp, R., ter Haar, F.: Shrec2007 3d shape retrieval contest. In: Technical Report, Department of Information and Computing Sciences, Utrecht University. (2007)

52. Jayanti, S., Kalyanaraman, Y., Iyer, N., Ramani, K.: Developing an engineering shape benchmark for cad models. Computer-Aided Design 38 (2006) 939-953 